\title{
PRODUCTION D'UN CHIRONOMIDE SEMIVOLTIN (CHIRONOMUS COMMUTATUS STR.) DANS LE LAC DE PORT-BIELH (PYRÉNEES CENTRALES)
}

\author{
par H. LAVILLLE.
}

La production annuelle nette de Ch. commutatus est évaluée, en 1971-7̇2 à $6,29 \mathrm{~kg} / \mathrm{PS} / \mathrm{ha}$, plaçant cette espèce au deuxième rang d'importance dans la production de la communauté benthique littori-profonde du lac. Le rapport $\mathrm{P} / \mathrm{B} \max =1,49$ confirme la stabilité de sa valeur, précédemment montrée chez plusieurs représentants du benthos, et, plus récemment, chez deux crustacés monocycliques du zooplaneton de ce même lac.

Plus du tiers $(36 \%)$ de la production est perdu pour l'écosystème lors de la nymphose $(30 \%)$ et de l'émergence des adultes $(6 \%)$.

Les truites (Salmo trutta fario $L$. ) utilisent $10,5 \%$ de la production de $C h$. commutatus par l'ingestion de nymphes au cours de leur ascension; cela représente $49 \mathrm{~kg} / \mathrm{PF}$ consommés pour les 10,5 ha de la zone $7-19 \mathrm{~m}$.

\section{Production of a semi-voltine chironomid,}

\section{Chironomus commutatus Str., in Lake Port-Bielh (Central Pyrenees).}

The net annual production of Ch. commutatus was estimated in 1971-72 as $6.29 \mathrm{~kg} / \mathrm{ha} / \mathrm{dry}$ weight, placing this species second in importance in the production of the littoral-profundal benthic commuty of the lake.

The value of the ratio $\mathrm{P} / \mathrm{Bmax}=1.49$ confirms the stability of this ratio, a stability which has been previously shown for several representatives of the benthos and more recently for two monocyclic species of Crustacea from the same lake.

More of one third $(36 \%)$ of the production is lost by the ecosystem during the pupation $(30 \%)$ and emergence of the adults $(6 \%)$.

Brown trout (Salmo trutta fario) utilise $10.5 \%$ of the production of $C h$. commutatus when they feed on the ascending pupae; this represents $49 \mathrm{~kg} /$ weth weight for the 10.5 ha of the zone between the depths of $7-19 \mathrm{~m}$.

\section{I. - INTRODUCTION}

Il n'existe aucune méthode globale, semblable à celles utilisées pour les organismes autotrophes, permettant d'évaluer la production des invertébrés dans les écosystèmes aquatiques. Celle-ci peut être calculée par sommation des résultats obtenus pour chaque

1. Labnratoire d'Hydrobiologie, Université Paul-Sabatier, 118, route de Narbonne, 31077, Toulouse Cedex. 
espèce. Les lacs de haute montagne se prêtent davantage à ce genre d'investigations, du fait de leur faible diversité spécifique.

Dans des travaux précédenis, nous avons estimé la production de deux Chironomides, Psectrocladius sordidellus et Zavrelimyia melanura (Laville 1972 b) et celle d'un mégaloptère Sialis lutaria (Giani et Laville 1973) abondants dans la zone littori-profonde (7 - 19 m) du lac de Port-Bielh. Chironomus commutatus, autre espèce abondante de la communauté benthique de cette zone, représentait au dégel $197240,5 \%$ de la biomasse des Chironomides et 26,5\% de celle du macrobenthos. Son cycle de développement sur deux ans, sa distribution bathymétrique et les modalités de la croissance des larves ont été mis en évidence précédemment (Laville $1972 \mathrm{~b}$ ).

Dans ce travail, nous nous proposons de compléter l'étude de la production secondaire benthique par l'évaluation de la production annuelle nette de Ch. communtatus.

\section{II. - DISTRIBUTICN, CYCLE DE DÉVELOPPEMENT ET BIOMASSE}

L'espèce étudiée est la plus abondante des deux espèces de Chironomus (Ch. commutatus et Ch. plumosus) qui peuplent la zone littori-profonde du lac de Port-Bielh. Ch. plumosus lui succède en profondeur et subsiste seule au-delà de $16 \mathrm{~m}$ ( fig. $1 \mathrm{~A})$.

Nous avons donné la description morphologique de la larve de Ch. commutatus dans un travail précédent (Laville 1972 a). De type thummi, elle se distingue aisément de celle de Ch. plumosus par l'absence d'expansions latérales au niveau du $10^{\circ}$ segment abdominal ainsi que par une taille moindre.

Le cycle de développement de Ch. commutatus s'effectue sur deux ans : les premières éclosions larvaires ont lieu en août et les larves atteignent le stade III à l'entrée de l'hiver; elle ont alors une longueur de 4 à $8 \mathrm{~mm}$ pour une largeur de capsule céphalique de 0,3 à $0,4 \mathrm{~mm}$. Le passage du stade III au stade IV (larve de 9 à $14 \mathrm{~mm}$ avec une capsule céphalique de $0,5 \therefore 0,67 \mathrm{~mm}$ de large) se fait au cours du deuxième été. Les larves passent un deuxième hiver sous la glace avant la nymphose, le début des émergences se produisant, selon les années, fin juillet-début août (Laville 1972 a).

La quasi-totalité de la croissance se produit à l'automne lorsque la température du fond atteint sa valeur maximale. Croissance et mortalité larvaires sont faibles en hiver, les conditions thermiques et d'oxygénation de la zone profonde, pendant la phase de gel, ralentissant considérablement l'activité métabolique des larves. Ce phénomène est tout à fait semblable à celui précédemment mis en évidence chez les larves de Psectrocladius sordidellus et Zavrelimyia melanura. Par suite, l'effectif des larves et leur biomasse 


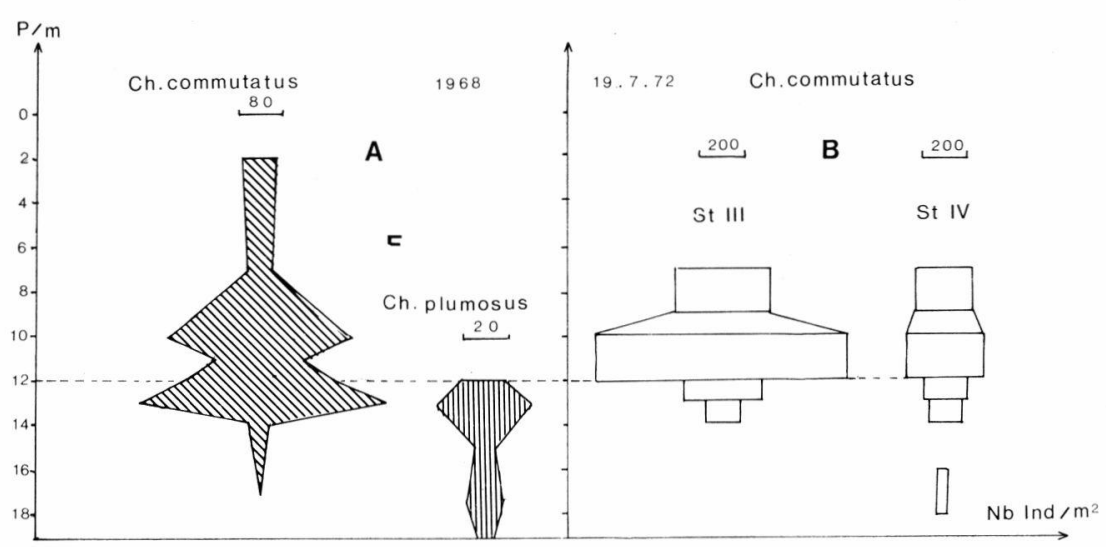

FIg. 1. - A) Densité des émergences de Chironomus commutatus et Chironomus plumosus pendant l'été 1968 en fonction de la profondeur.

B) Distribution bathymétrique des larves aux stades III et IV de Ch. commutatus d'après les prélèvements du 19 juillet 1972 .

varient peu au cours des 7 mois que dure le gel. Début juillet, la biomasse larvaire de Ch. commutatus atteint encore $94 \%$ de celle évaluée le 15 novembre.

On assiste le plus fréquemment chez les larves de Chironomides à un enfoncement progressif aux derniers stades du développement. Ce phénomène a été mis en évidence, notamment par Wülker (1961) chez Sergentia coracina dans un lac de Forêt-Noire, le Titisee $(845 \mathrm{~m})$, profond de $39 \mathrm{~m}$ et par Thut (1969) chez Procladius culiciformis du lac Washington. C'est aussi le cas de Ch. commutatus dont les larves IV s'avèrent plus eurybathes : leur distribution bathymétrique est plus uniforme dans les zones littoriprofonde que celle des stades III plutôt concentrés entre 10 et $13 \mathrm{~m}$ (fig. $1 \mathrm{~B})$.

\section{III. - PRODUC'TION}

Les évaluations de l'abondance larvaire au dégel lors de trois années d'observation $(1968,69,72)$ révèlent une certaine stabilité de la population de Ch. commutatus. La densité moyenne dans la zone littori-profonde est de 668 larves/ $\mathbf{m}^{2}$, l'écart des valeurs extrêmes ne dépassant pas $9 \%$ :

$$
\begin{aligned}
4-7-1968 & : 675 \text { larves } / \mathrm{m}^{2} ; \\
5-7-1969 & : 609 \text { larves } / \mathrm{m}^{2} ; \\
19-7-1972 & : 721 \text { larves } / \mathrm{m}^{2} .
\end{aligned}
$$

Cette stabilité s'applique à tous les stades larvaires : elle permet donc d'estimer, avec un minimum d'échantillons, l'évolution numérique de la population au cours de son développement. 


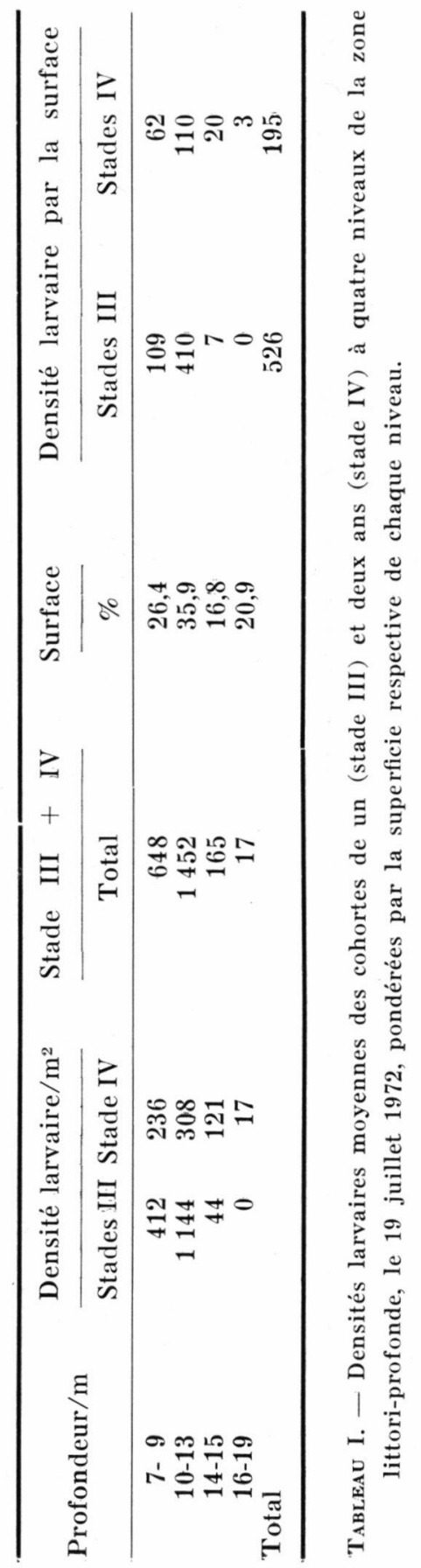




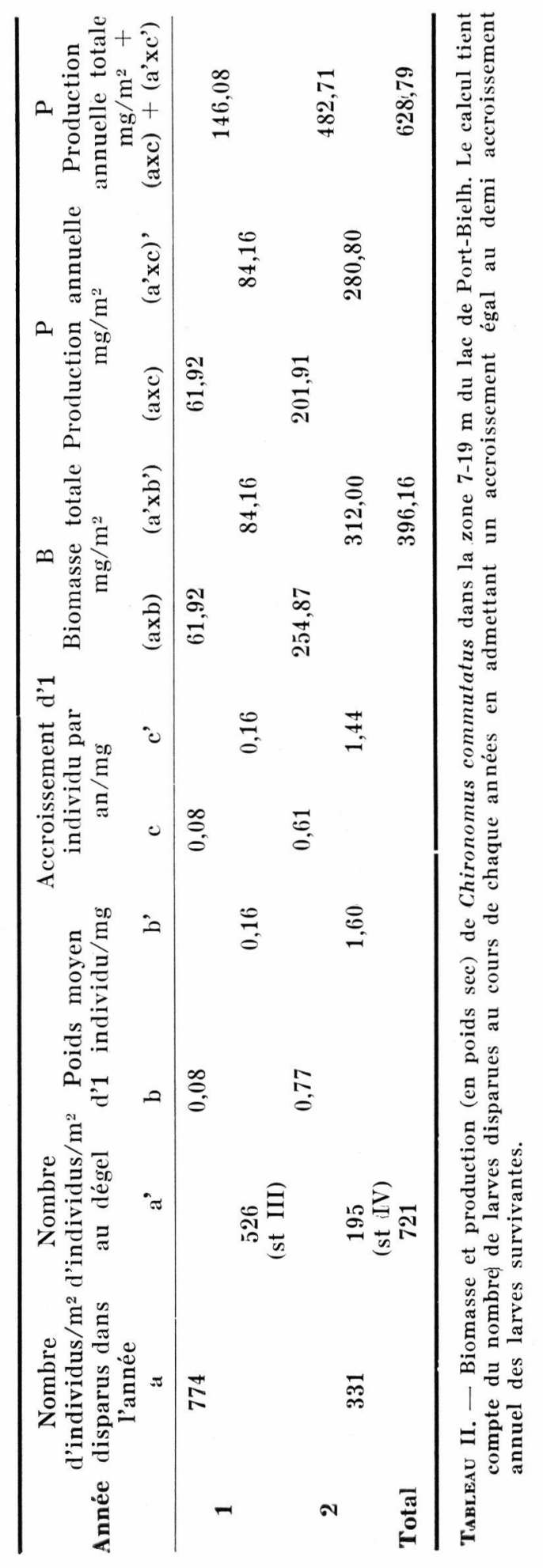


31 dragues de type Ekman ont été prélevées le 19 juillet 1972, avant le début des émergences. Elles se répartissent comme suit :

$$
\begin{array}{r}
8 \text { entre } 7 \text { et } 9 \text { mètres; } \\
11 \text { entre } 10 \text { et } 13 \text { mètres; } \\
4 \text { entre } 14 \text { et } 15 \text { mètres; } \\
8 \text { entre } 16 \text { et } 19 \text { mètres. }
\end{array}
$$

Ces prélèvements ont été lavés sur place au travers d'un filet fin à vide de mailles de $280 \mu$ et les larves triées au laboratoire à la loupe binoculaire, séparées par stade et mesurées. La relation taille-poids établie précédemment (Laville $1972 \mathrm{~b}$ ) a été utilisée pour déterminer le poids sec.

Le tableau I récapitule la densité respective des deux cohortes dans les quatre zones de prélèvement. Ces valeurs, pondérées par la superficie de chacune de ces zones donnent la densité larvaire moyenne de la zone littori-profonde : $73 \%$ des larves appartiennent à la cohorte de la première année, $27 \%$ à celle de la seconde année.

La production de $C h$. commutatus a été estimée par une méhode analogue à celle utilisée pour Sialis lutaria (Giani et Laville 1973) et qui est en fait une variante de la formule de Boysen-Jensen (1919):

$$
\mathrm{P}=\mathrm{N} 2(\mathrm{~W} 2-\mathrm{W} 1)+\mathrm{N} 1-\mathrm{N} 2 \frac{\mathrm{W} 2-\mathrm{W} 1}{2}
$$

La première partie de la formule donne la production de chaque cohorte connaissant l'accroissement pondéral des individus pendant l'année. La production des animaux morts est donnée par la seconde partie de la formule el correspond aux larves de la cohorte disparues au cours de l'année. Si l'on admet une courbe de mortalité de la forme d'une exponentielle décroissante, on peut estimer à $780 / \mathrm{m}^{2}$ le nombre de larves de première année perdues entre la ponte et le mois de juillet. Compte tenu de leur faible poids individuel, cette perte ne représente en fait qu'une portion restreinte de la production totale.

La production annuelle des deux cohortes est exprimée en poids sec dans le tableau II : elle atteint $629 \mathrm{mg} / \mathrm{m}^{2}$ soit $6,29 \mathrm{~kg} / \mathrm{ha}$. Près de $77 \%$ de cette production sont assurés par les larves de deuxième année ; $23 \%$ seulement représentent la production au cours de la première année de développement. La production des animaux disparus par mortalité naturelle ou prédation représente $42 \%$ de la production totale dont $10 \%$ seulement sont le fait des larves de la première cohorte.

La quantité moyenne de matière vivante perdue lors de l'émergence des imagos de $C h$. commutatus a été évaluée à partir des récoltes de nymphes et d'adultes dans 15 pièges à émergence placés 
entre 7 et $13 \mathrm{~m}$ en $1966,67,68$ et 70 . Le nombre moyen d'émergences obtenues dans celle zone est de 146 ind./ $\mathrm{m}^{2} / \mathrm{an}$. Comple tenu de la distribution des larves au stade IV dans le fond et de la répartition des pièges à émorgences, on peul estimer que pour l'ensemble de la zone littori-profonde le nombre moyen d'émergences $/ \mathrm{m}^{2} /$ an avoisine 93 imagos. Ainsi, sur les 195 larves proches de la nymphose recensées en juillet, $48 \%$ émergent.

Le poids sec moyen des imagos, obtenu après 15 heures de séchage à $105^{\circ} \mathrm{C}$ est de $0,3 \mathrm{mg}$ pour les ô et de $0,55 \mathrm{mg}$ pour les o. Etanl donné l'évolution du sex-ratio avec la profondeur (1,04 à $10 \mathrm{~m} ; 1,46$ entre 11 et $13 \mathrm{~m}$ : Laville $1972 \mathrm{~b}$ ) on peul admettre un rapport moyen de 1,25 soil 52 s pour 41 \%. Le poids des émergences correspond done à une biomasse de $38,1 \mathrm{mg} / \mathrm{PS} / \mathrm{m}^{2} / \mathrm{an}$. Cette masse ne représenle en fail que $6 \%$ de la production annuelle de Ch. commutatus. Ce pourcentage est très voisin de celui calculé pour $P$ s. sordidellus $(8 \%)$ mais légèrement plus faible que pour $Z$. melanura $(15 \%)$ dont l'effectif des larves est plus difficile à évaluer du faí de la mobilité de cette forme essenliellement carnassière (Laville 1972 b). Des rapports émergence/production, très variables, sont cités dans la litlérature. Ainsi, Jonasson (1972) évalue cette perte à $52 \%$ de la production de Chironomus anthracinus dans le lac Esrom. En milieu lotique, Neveu (1973) estime que les adultes de plusieurs espèces de Simulium représentent 20 à $25 \%$ de la production larvaire. Par contre, nos résultats rejoignent ceux de Hall, Cooper et Werner (1970) qui ont estimé que les émergences de Chironomus tentans représentaient $7,9 \pm 1,9 \%$ de la biomasse produite.

Calculé à partir de la biomasse du 19 juillet, le rapport $\mathbf{P} / \mathbf{B}$ $=1,58$. L'utilisation des courbes de croissance (Laville $1972 \mathrm{~b}$ ) et de mortalité permet d'estimer que la biomasse larvaire atteint son maximum $\left(422 \mathrm{mg} / \mathrm{PS} / \mathrm{m}^{2}\right)$ à l'entréc de l'hiver. Le rapport avoisine alors 1,49 , valeur très proche de celles précédemment trouvées pour Ps. sordidellus, $Z$. melanura et Sialis lutaria. L'étude de la production d'un Trichoptère, Micrasema difficile, dans un ruisseau d'altitude des Pyrénées (Décamps el Laffont 1974) et plus récemment celle des Crustacés planctoniques du lac de Port-Bielh (Rey el Capblancq 1975) ont mis en évidence des rapports $\mathrm{P} / \mathrm{B}$ max du même ordre. La stabilité de celte valeur reflète très vraisemblablement l'analogie de développement des invertébrés d'altitude que détermine l'alternance de deux saisons (tableau III).

La stabilité de ce rapport permet ainsi d'estimer la production des larves d'insecles (Chironomides + Sialis) du lac de Port-Bielh à $2,035 \mathrm{~g} / \mathrm{m}^{2} / \mathrm{an} / \mathrm{PS}$. Les 4 espèces dominantes représentent $\mathbf{7 6 , 6} \%$ de cette production totale : $19,5 \%$ sont assurés par Ch. commutatus.

Les valeurs de la production secondaire des espèces dominantes 


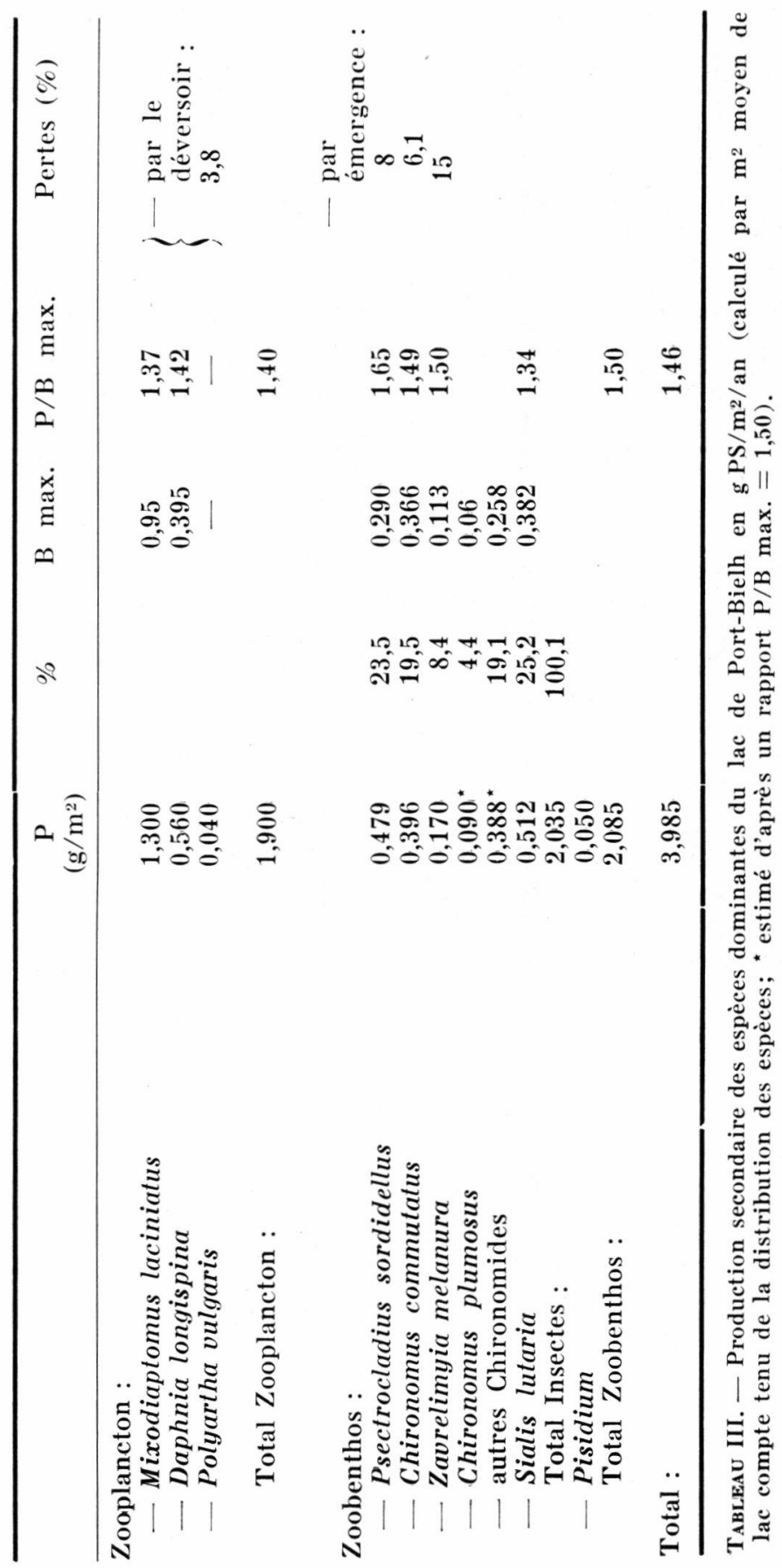


du lac de Port-Bielh obtenues en tenant compte de la distribution respective de chaque espèce sont récapitulées sur le tableau III.

\section{IV. - DISCUSSION}

La production des larves d'insectes aquatiques doit être considérée sous deux aspects différents selon qu'elle quitte ou reste dans l'écosystème.

Une part importante de la production larvaire est utilisée pour couvrir les besoins métaboliques, particulièrement intenses à la nymphose. La diminution de poids enregistrée entre la larve IV de Ch. commutatus et la nymphe correspond à une dépense énergétique ; elle représente $29,5 \%$ de la production annuelle de cette espèce. Ainsi, avec les imagos qui émergent, plus de $35 \%$ de la production est perdu par l'écosystème. Cette proportion correspond vraisemblablement à la perte maximum envisageable puisque la part d'exuvies nymphales susceptible de quitter le lac par le déversoir doit être compensée par l'apport issu de la ponte des adultes.

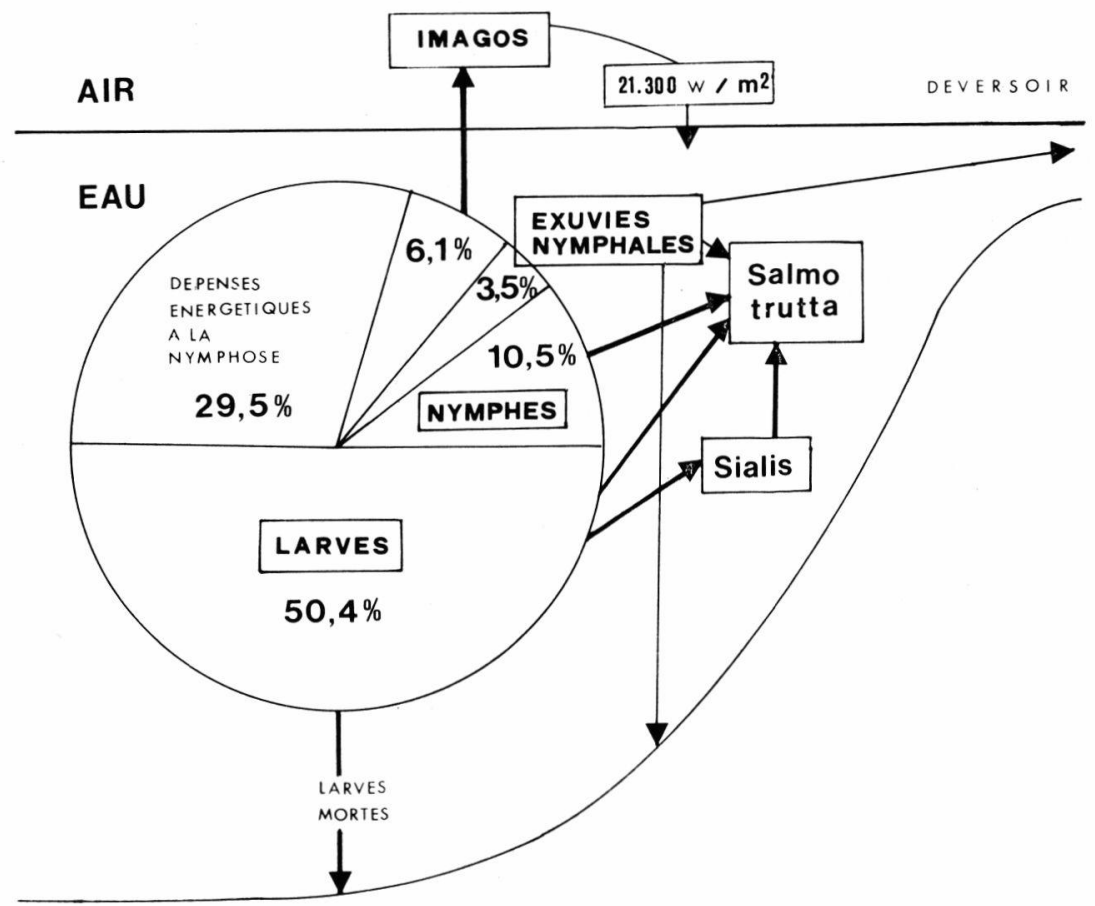

FIg. 2. - Diagramme simplifié du cycle de la matière produite par la population de Chironomus commutatus du lac de Port-Bielh. 
$\mathrm{Si}$ on suppose que toutes les femelles qui émergent sont fécondées et pondent dans le lac à raison de 520 oeufs/individus, on peut estimer à 21300 le nombre d'œufs pondus par $\mathbf{m}^{2}$ de lac.

Près des $2 / 3(64,4 \%)$ de la production ne quittent pas le lac et sont théoriquement disponibles pour les niveaux trophiques suivants (fig. 2). En fait, une analyse plus détaillée montre qu'une partie seulement de la biomasse est exploitée, la prédation dépendant du stade de développement des proies et du comportement des prédateurs. Ainsi, dans l'exemple présent, les larves âgées de Sialis lutaria consomment des stades larvaires III et IV de Ch. commutatus (Giani et Laville 1973). La prédation des truites s'exerce essentiellement sur les nymphes : l'analyse de 26 contenus stomacaux de truites capturées en été révèle $93 \%$ de nymphes et seulement $7 \%$ de larves IV sur les $162 \mathrm{Ch}$. commutatus recensés. La stabilité des populations entre le gel et le dégel semble indiquer que la prédation hivernale au niveau larvaire est faible. Dans un lac alpin, le Vorderer Finstertaler See, Pechlaner et al. (1972) ont noté la présence de faune benthique seule dans les contenus stomacaux des Salmonides capturés pendant l'hiver. Cependant, les travaux de Elliott (1973 et 1975) ont montré que le nombre de repas journaliers de même que la quantité de nourriture prise par repas diminuent fortement aux basses températures chez la truite (S. trutta).

Si on considère que la différence entre le nombre d'émergences et de larves au dernier slade correspond à la prédation des seules truites sur les nymphes lors de leur ascension, la quantité de nourriture consommée correspond à $6,96 \mathrm{~kg} / \mathrm{PS} / \mathrm{ha}$ soit $10,5 \%$ de la production nette de $C h$. commutatus. La prédation directe au niveau larvaire et par l'intermédiaire des larves de Sialis peut augmenter légèrement ce pourcentage mais ne semble pas devoir le modifier de façon importante.

Le rendement correspondant à l'utilisation de la production benthique par les poissons dépend donc en grande partie du comportement de prise de nourriture el de l'accessibilité de cette dernière. Aussi paraît-il difficile de prévoir la cappacité productive en poissons à partir des seules mesures de la biomasse ou de la productivité des niveaux trophiques précédents.

\section{TRAVAUX CITES}

Boysen-JENSEN (P.). 1919. - Valuation of the limfjord. I. Studies on the fish food on the I,imfjord 1909-1917. Rep. Dan. biol. Sin., $26: 1-44$.

DÉCAMPS (H.) et LAFon' (M.). 1974. - Cycles vitaux et production des Micrasema pyrénéennes dans les mousses d'eau courante. Annls Limnol., 10 (1) : 1-32. 
Elliott (J. M.). 1973. - The food of Brown and Rainbow Trout (Salmo trutta and $S$. gairdneri) in relation to the abundance of drifting invertebrates in a mountain stream. Oecologia, 12 : 329-347.

Elliott (J. M.). 1975. - Weight of food and time required to satiate brown trout, Salmo trutta L. Freswat. Biol., 5 : 51-64.

Giani (N.) et Laville (H.). 1973. - Cycle biologique et production de Sialis lutaria L. (Mégaloptère) dans le lac de Port-Bielh (Pyrénées centrales). Annls Limnol., 9 : 45-61.

Jonasson (P. M.). 1972. - Ecology and production of the profundal benthos in relation to phytoplancton in Lake Esrom. Oikos, suppl. $14: 1-148$.

Laville (H.). 1972a. - Recherches sur les Chironomides (Diptera) lacustres du massif de Néouvielle (Hautes-Pyrénées). I. Systématique, écologie, phénologie. Annls Limnol., (1971), 7 (2) : 173-332.

Laville (H.). 1972b. - Recherches sur les Chironomides (Diptera) lacustres du massif de Néouvielle (Hautes-Pyrénées). II. Communautés et production. Annls Limnol., (1971), 7 (3) : 335-414.

Neveu (A.). 1973. - Estimation de la production de populations larvaires du genre Simulium (Diptera, Nematocera). Ann. Hydrobiol., 4 (2) : 183-199.

Rey et Capblance (J.). 1975). - Dynamique des populations et production du zooplancton du lac de Port-Bielh (Pyrénées centrales). Annls Limnol., 11 (1) :

Pechlaner (R.), Bretschio (G.), Gollmann (P.), Pfeifer (H.), Tilzer (M.), and Weissenbach (H. P.). 1972. - The production processes in two high-mountain lakes (Vorderer and Hinterer Finstertaler See, Kühtai, Austria). Proc. IBP-UNESCO, Symposium on Productivity Problems of Freshwaters, Kazimierz-Dolny, Poland, May $6-12,1970: 239-269$.

Thut (N. R.). 1969. - A study of the profundal bottom fauna Lake Washington. Ecol. monographs, 39 (1) : 79-100.

WüLKer (W.). 1961. - Lebenszyklus und Vertikalverteilung der Chironomidae (Dipt.) Sergentia coracina Zett. im Titisee. Verh. int. Ver, Limn., 14 : 962-967. 